\title{
CROSSING SIMPLE RESONANCES
}

\author{
T. Collins
}

August 1986 


\title{
Croseing Simple Resonances
}

\author{
Tom Collins \\ Fermilab \\ August 6, 1986
}

\begin{abstract}
A simple criterion governs the beam distortion and/or loss of protons on a fast resonance crossing. Results from numerical integrations are illustrated for simple sextupole, octupole, and 10-pole resonances.
\end{abstract}


It is well-known that if one crosses a resonance quickly then no protons are lost. The question is how quick is quick. The following results are for simple resonances with no tune-shifts, polynomial amplitude expressions, or other complications.

If one starts well below the resonance point, at a tune $-\delta$, with protons in beta phase-space represented by a disk of amplitude ao and then tunes to the resonance adiabatically, the protons will fill the separatrix at $-\delta_{0}$ where

$$
\begin{array}{ll}
a_{0}=.6430\left(8 \pi \delta_{0} / A\right) & \text { sextupole } \\
a_{0}^{2}=.5611\left(16 \pi \delta_{0} / A\right) & \text { octupole } \\
a_{0}{ }^{3}=.5225\left(32 \pi \delta_{0} / A\right) & 10 \text {-pole }
\end{array}
$$

and $A$ is the usual driving term. Any two of $a_{0}, \delta_{0}, A$ defines the resonance width. The same size of separatrix occurs at $+\delta_{0}$, but it is rotated half-way.

I define $n_{0}$ as the number of turns to change the tune by $\delta_{0}$, that is $d \delta / d n=\delta_{0} / n_{0}$. 2no is needed to cross the full resonance width for ao. We will find that the quantity $\left[\delta_{0} n_{0}\right]$ completely determines the beam behaviour.

When we start well below and approach resonance at a finite rate, the early beam shapes are not appreciably different from the adiabatic case. The separatrix is much larger than the beam and as it shrinks the beam is distorted from a circle in a predictable way. However when the separatrix closes on the beam, deviation from the adiabatic shape is significant and protons which are left outside the separatrix have various behaviours. Some protons are near the outgoing branch of the separatrix and start to increase their amplitude, others are near the ingoing branch and follow the separatrix towards smaller amplitudes, while many simply re-adjust their phase.

The separatrix passes through zero and then grows but the branches are shifted to a new position so the motion of the external protons is complicated, for example some protons heading out reverse direction and head in. The outward sweeping separatrix gobbles up many of the protons and these subsequently settle down to circular phase trajectories but at new amplitudes. There are some however near the new outgoing branch and these are involved in a new version of a classic footrace.

Achilles, the separatrist, has a running start. Somewhat down the road are some inverse-turtles, just getting moving, which Achilles is trying to pass. Now one must not confuse these turtles with the Old-world Xenon Tortoise, a rather dull creature that always moves half as far (well maybe not always). The American inverse-turtle, which has 3, 4, or 5 legs (see illustration), moves twice as far, and so we have a much more interesting race, a kind of half a 
drag race where the inverse-turtle escapes if it can match Achilles speed before being passed. Do not bet on whether any inverse-turtles escape, the

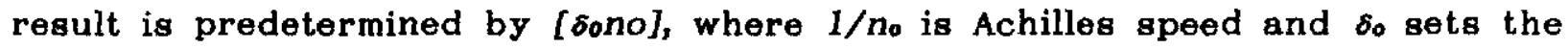
inverse-turtle's acceleration, however one can bet on which inverse-turtles escape because that is not known without actually running a race.

The diagrams show the development of a race where proton $X$ escapes but it close companions - two squares - do not.

\section{A Little Theory}

Let me write the equations for the sextupole case, which I express as differential equations (as I usually do)

$$
\begin{aligned}
d a / d n & =(A / 4) a^{2} \sin 3 \varphi \quad n=\text { turns } \\
d(3 \varphi) / d n & =3(A / 4) a \cos 3 \varphi+6 \pi \delta(n) \quad \delta(n)=n \delta_{0} / n_{0}
\end{aligned}
$$

in which the only new thing is that $\delta$ changes with $n$ (I use $n=0$ for the resonance point, and start with a large negative $n$ ).

Now we make some simple substitutions. Let $u=a / a_{0}$ and $z=n / n_{0}$ then

$$
\begin{aligned}
d u / d z & =C\left[\delta_{0} n_{0}\right] u^{2} \sin 3 \varphi \quad C=2 \pi(.6430) \\
d(3 \varphi) / d z & =3 C\left[\delta_{0} n_{0}\right] u \cos 3 \varphi+6 \pi\left[\delta_{0} n_{0}\right] z
\end{aligned}
$$

and it is clear that [ $\left.\delta_{0} n_{0}\right]$ completely determines the solution. We start with $u=1$ and $\varphi=\varphi_{0}$ far from resonance - a large negative $z$ - and integrate numerically. This is a slow process and one can avoid much of the predictable portion by starting at $z=-10$ and setting

$$
u=1-(C / 6 \pi z) \cos 3 \varphi_{0} \quad \varphi=\varphi_{0}+(C / 6 \pi z) \sin 3 \varphi_{0}
$$

which is the normal beam distortion (from a circle) when far from resonance. This correction is too small to show on the diagrams but it is necessary to avoid confusion near the limit for beam loss. An inverse distortion at $z=+10$ has been applied before plotting in lieu of a more extended numerical integration. Each point on the diagrams represents a different $\varphi_{0}$ and requires considerable arithmetic. 


\section{Discussion}

Results are given for sextupole, octupole, and decupole resonances, one page for each. The upper diagram is for [ $\left.\delta_{0} n_{0}\right]$ just slightly below the limit for beam loss, (ndo on the digrams is $\left[\delta_{0} n_{0}\right]$ ). The shape of the diagram is significant but the orientation is not because these shapes are far from resonance and are rotating rapidly. Note that the phase-space area has been conserved, and so has the original uniform density.

The limiting values for no beam loss are

$$
\begin{array}{rlrl}
{\left[\delta_{\circ} n_{0}\right]} & <.052 & \text { for sextupole } \\
& <.0285 \text { for octupole, and } \\
& <.0197 \text { for 10-pole, }
\end{array}
$$

however the use of differential equations becomes dubious if no is only a few turns, particularly if the multipole distribution is lumpy.

We can write $\delta^{\prime}=d \delta / d n$ then

$$
\begin{gathered}
\delta^{\prime}=\delta_{0} / n_{0}=\delta_{0}^{2} /\left[\delta_{0} n_{0}\right] \\
\text { and }\left[\delta_{0} n_{0}\right] \sim a_{0}^{2} / \delta^{\prime}, \sim a_{0} 4 / \delta^{\prime}, \sim a_{0} \delta / \delta^{\prime}
\end{gathered}
$$

for 6-, 8-, and 10-pole respectively, a strong amplitude dependence.

An initial sharp edged uniform disk of particles creates the most striking changes in beam shape. An initially gaussian beam that loses a few protons on resonance crossing is almost imperceptably altered. If one plots the usual Raleigh distribution $P(u) d u$ one finds a long but very sparse tail. The rms is increased slightly.

I have jugt set a trap, one that I once jumped into with both feet. Suppose that there is a small tune-shift with amplitude, for example a change of $\nu_{x}$ with $y^{2}$. If we wait a while after crossing then the pattern will be smoothed into circular symmetry. We can cross again and again increase the rms (by about the same amount) and repeat at infrequent intervals to allow for smoothing. And now we have it, the Holy Grail, steady emittance growth!

There is something wrong, of course. In the first place one notices that the central density of the distribution is never changed, and the rms does change so we don't have a gaussian anymore. This starts us on a horribly long calculation tracing the details of the change in distribution, both in crossing the resonance and in the waiting period. One finds an initial small loss, but the amount of loss diminishes rapidly with subsequent crossings as does the growth in the rms. 
Actually all of our equations preserve phase-space. If I start with a small differential volume in six-dimensional phase space containing a few particles, then this bit of space may be twisted and distorted but its volume remains the same and it contains the very same particles. The only thing that multiple crossings do is to insert a thread of empty space into the outer part of the beam, the central core is untouched. This is only our old "effective but not actual" phase dilution, similar to an injection mismatch but not nearly as effective, not the Holy Grail!

The origin of the problem is instructive. The first evaluation of a change in the rms was legitimate, the second and subsequent evaluations were not for in order to simply add the changes it is necessary that the particles have a randomized phase, which is not true. It is well known that if we can introduce a small truly random process then we can obtain true emittance growth, and that resonance type motion makes very effective use of a little randomness. Thus we managed to insert a random effect in a well hidden way where the word "random" never appears! (A much less subtle way is to insert "diffusion" without noting that diffusion is a random process.)

In practice then one can say that if a normally distributed beam crosses a resonance with very small loss then it can cross a number of times without perceptable degradation. In all cases the "core" density is unchanged. The criterion would be that the limiting [ $\left.\delta_{0} n_{0}\right]$ would correspond to an amplitude of say $3 \sigma$, where no is the number of turns to cross the adiabatic half-width $\delta_{0}$. 
<smiles>CC(C)(C)C(C)(C)C</smiles> 

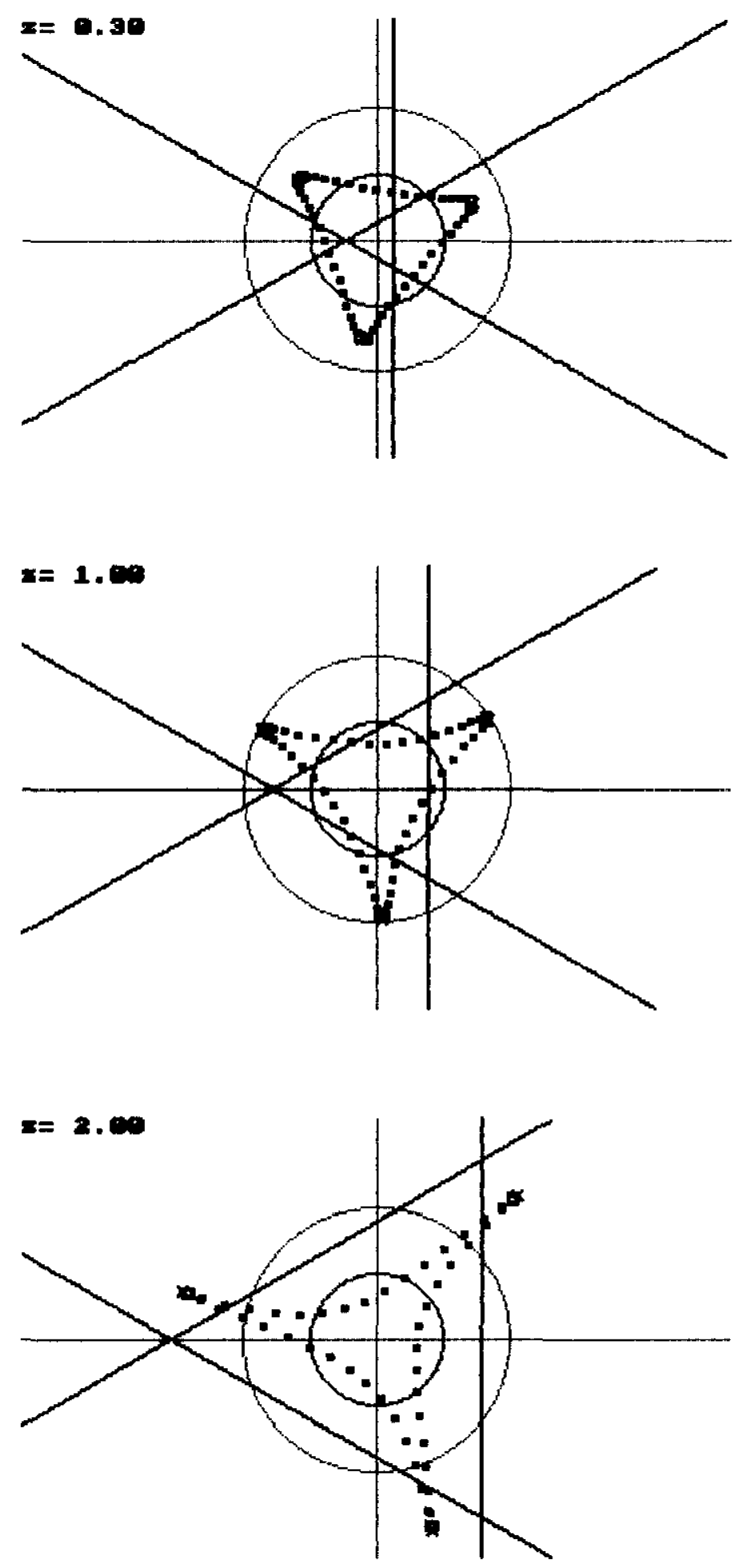

Sext. crossung, contisued 
8.
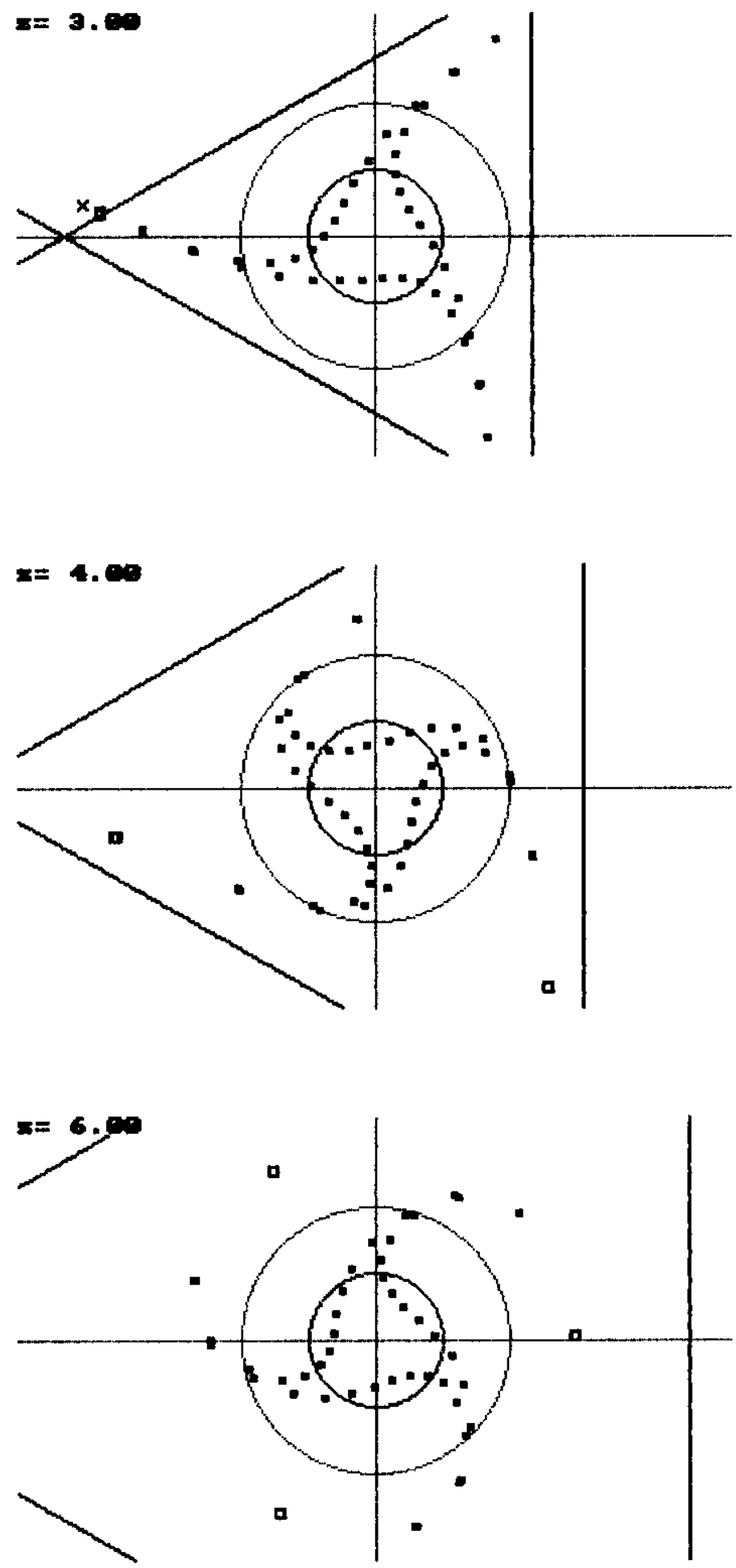

Sextupole reoonance crosoing completsd, $X$ is lost, two t's coerlap 
9.
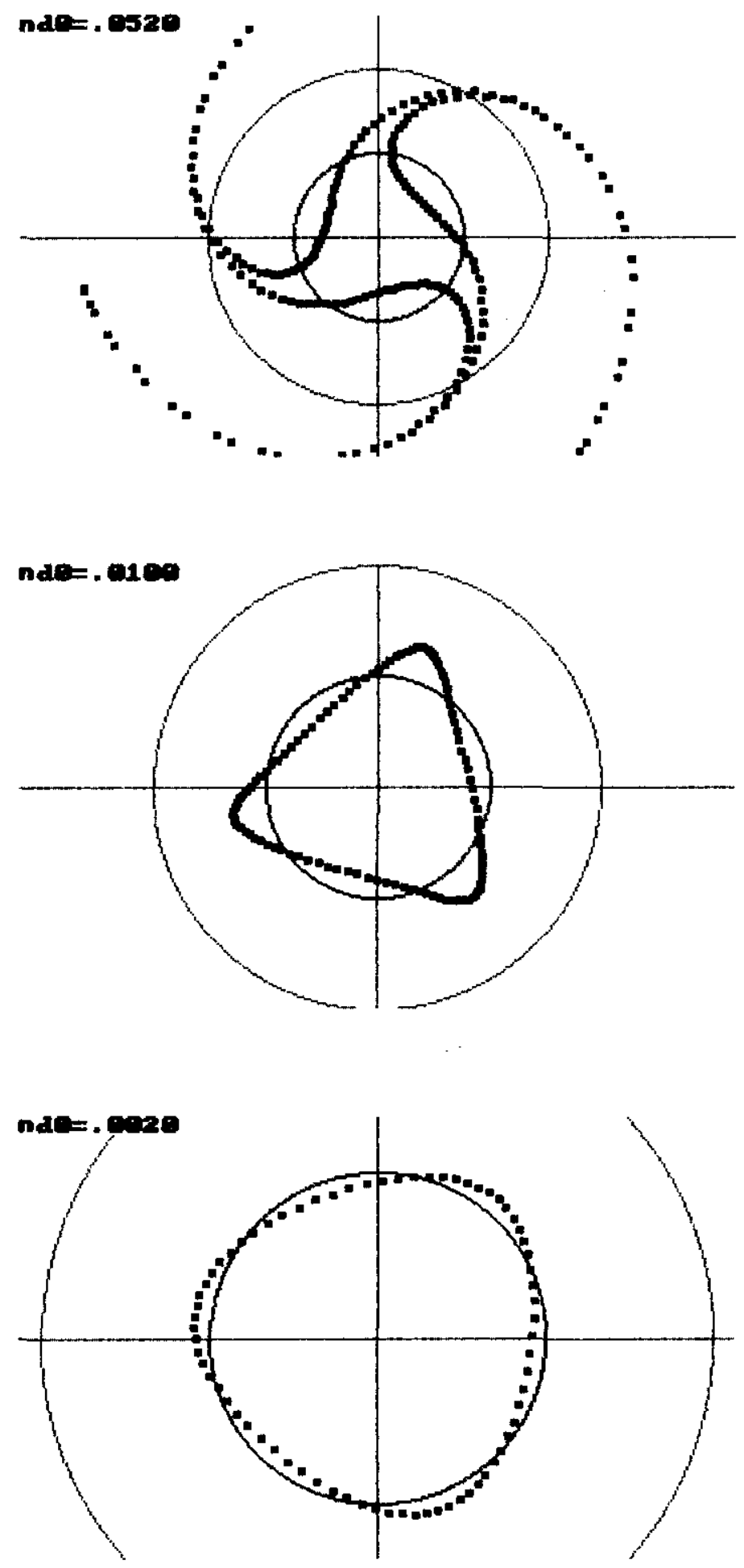

Sextupole nesonance. Beaws shafor after rnocoing 
10.
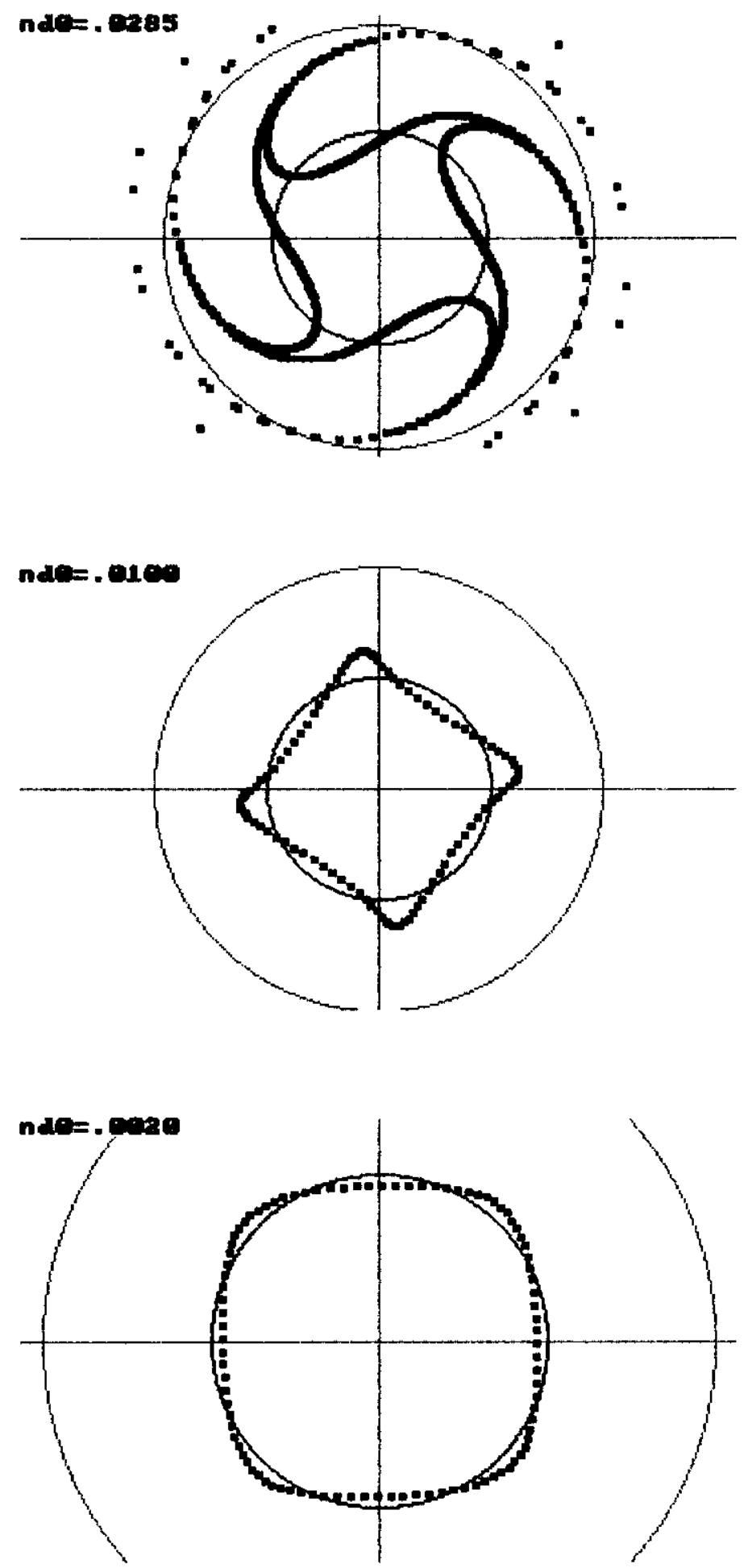

Octupole neo. crosoing 

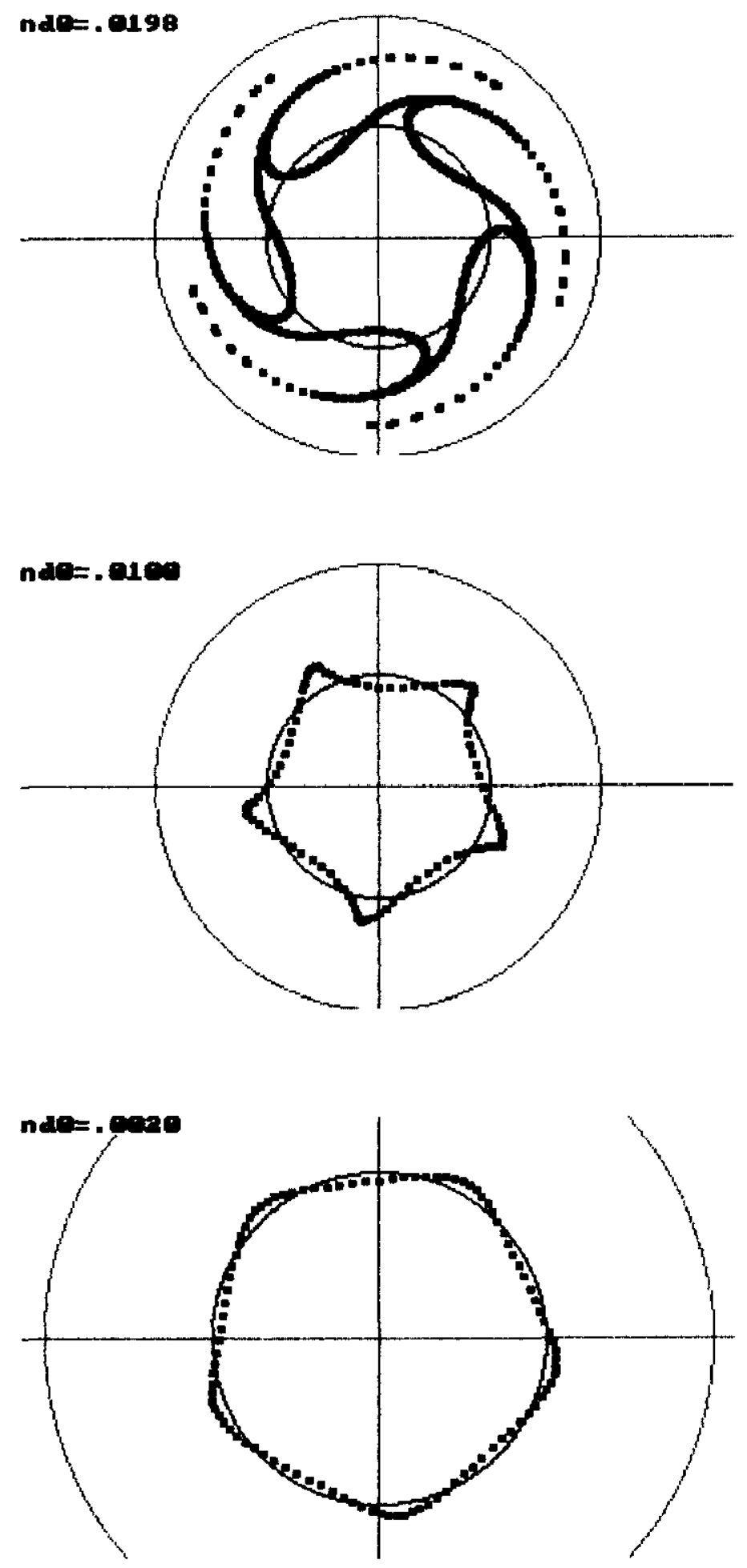

10-pole sesonance eroosing 